Insights into myofibroblasts and their adivation in sderoderma: opportunities for therapy?

\title{
Authors
}

Dafni A. Gyftaki-Venieri, David J. Abraham, Markella Ponticos

\section{Authors Affiliations}

Centre for Rheumatology and Connective Tissue Diseases, Research Department of Inflammation,

Division of Medicine, University College London, London, UK

\section{Author of correspondence}

Name: Dr Markella Ponticos (PhD)

Address: Centre For Rheumatology and Connective Tissue Diseases,

University College London (Royal Free Campus)

Rowland Hill Street,

London, NW3 2PF

United Kingdom

Telephone Number: +44 (0)207 7940500 ext 38546

Email address: mponticos@ud.ac.uk 


\section{Abstract}

\section{Purpose of review}

The persistence of myofibroblasts is a key feature of fibrosis and in fibrotic diseases including scleroderma. This review evaluates the emerging concepts of the origins and cell populations that contribute to myofibroblasts and the molecular mechanisms that govern phenotypic conversion, that highlight opportunities for new interventional treatments in scleroderma.

\section{Recent Findings}

Studies have defined heterogeneity in fibroblast-like cells that can develop into myofibroblast in normal wound healing, scarring and fibrosis. Characterising these distinct cell populations and their behaviour has been a key focus. In addition, the overarching impact of epigenetic regulation of genes associated with inflammatory responses, cell signalling and cell communication and the ECM has provided important insights into the formation of myofibroblast and their function. Important new studies indude investigations into the relationship between inflammation and myofibroblast production and further evidence has been gathered which reveal the importance of ECM microenvironment, biomechanical sensing and mechanotransduction.

\section{Summary}

This review highlights our current understanding and outlines the increasing complexity of the biological processes that leads to the appearance of the myofibroblast in normal functions and in diseased tissues. We also focus on areas of special interest in particular, studies which have therapeutic potential in fibrosis and scleroderma.

\section{Keywords}

Myofibroblasts, Epigenetic, Fibroblast Heterogeneity, Fibrosis, Scleroderma 


\section{Introduction}

The myofibroblast is an activated mesenchymal cell-type associated with normal tissue repair as well as scarring and fibrosis and is characterised by collagen secretion and the expression of $\alpha$-smooth muscle actin ( $\alpha \mathrm{SMA})$. $\alpha \mathrm{SMA}$ form prominent filament bundles known as stress fibres which reinforce the cell cytoskeleton and promote contractile force generation (1).

Persistent activation to myofibroblast is one of the key aspects of pathological fibrosis that distinguishes it from controlled wound healing during tissue repair after acute injury. The persistent response of myofibroblasts to soluble factors, abnormal extracellular matrix (ECM) cues and dysregulated cellular communication, can lead to further matrix deposition and fibrosis (2). Many have argued that inhibiting myofibroblast formation may represent an effective way to attenuate the fibrotic process or even halt or reverse established fibrosis. Therefore, targeting the formation, function and survival of myofibroblast has been the objective of many studies in fibrotic diseases such as scleroderma (3). Scleroderma is an inflammatory, autoimmune, fibrotic connective tissue disease that has long been associated with persistent activation of fibroblasts and other progenitor cell types towards the myofibroblasts phenotype via the action of Transforming Growth Factor $\beta$ (TGF $\beta$ ) and excessive deposition of ECM such as collagen and fibronectin (4).

A complex biological network of processes and factors is known to affect myofibroblast formation and myofibroblast persistence within tissues (Figure 1). In the past, the best described pathways to myofibroblast production were centred on activation by TGF $\beta$ and/or Platelet Derived Growth factor (PDGF). In recent years, it has emerged that other processes, such epigenetic regulation, inflammation, inter and intra-cellular communication/miscommunication and alteration in biomechanics and the ECM, are critical in normal and disease-associated myofibroblast differentiation. These factors form intricate and elaborate pathways that underpin regulatory control. Epigenetics influences act on the ECM and the altered stiffness and associated forces it presents, is exerted on resident fibroblasts, as well as on inflammatory signals and their effect on 
fibroblast signalling and cellular functions such as differentiation, proliferation, migration and survival. An additional facet of an already complex picture, is the identification of fibroblast heterogeneity. Resident fibroblast subpopulations with varying functionality are likely to have a profound influence on the morphological and functional heterogeneity of myofibroblasts in both normal and diseased tissues. In this review, we highlight some of the more recent findings and new concepts under investigation, as well as discuss the opportunities for novel therapies that arise from these studies.

\section{Fibroblast Origins and Heterogeneity}

The notion of fibroblast heterogeneity and the presence of subpopulation in tissues is not new. Fibroblast diversity has preoccupied researchers of wound healing and various fibrotic diseases from as early as the 1980s where work by Kaus Bayreuther (5) identified eleven fibroblast-like cell types in human skin. Advances in cell phenotyping and genetic markers for lineage tracking has greater increased our appreciation of fibroblast subsets. In addition, in order to understand the fibroblast/myofibroblast relationship and functionality, researchers have looked to the origins of these cells in tissues (6). Potential sources of myofibroblast cell types include the resident tissue fibroblasts, epithelial cells, bone marrow derived progenitor stem cells, endothelial cells, pericytes, smooth muscle cells, resident mesenchymal progenitors, and adipocytes $(1,7,8)$.

In 2013, Driskell et al presented a comprehensive mouse skin study which supported the idea that resident tissue fibroblasts may be a heterogeneous group of cells with specialised functions that regulate the ECM and coordinate neighbouring cell function (9). These authors demonstrated that spatial distribution in the upper and lower dermis correlates with distinct lineages. Elegant cell tracking studies using the developmental homeobox transcription factor engrailed 1 also identified an intrinsic fibroblast lineage, exhibiting surface expression of CD26 (DPP4; dipeptidyl peptidase-4), and responsible for healing and fibrosis, when explored in in vivo models (10). There is now good evidence suggesting that DPP4(CD26) can regulate inflammatory responses, defines a pro-fibrotic 
fibroblast population and suggests that DPP4(CD26) inhibition may be a potential treatment of fibrosis in the skin and other organs (11).

Recent studies have also identified distinct human fibroblast lineages and a variety of subpopulations that may result in pro-fibrotic phenotypes. Using single-cell RNA sequencing on healthy skin fibroblasts, Tabib et al identified two major fibroblast populations defined by expression of SFRP2/DPP4(CD26) and FMO1/LSP1 and 5 minor populations with different functional roles in inflammation and regulation of ECM deposition (12). A different study exploring human dermal fibroblasts, identified at least four distinct subpopulation were identified based on of lin-CD90, CD39, RGS5 and DPP4(CD26), each with distinct morphology and cellular function in terms of WNT signalling and interferon responsiveness (13).

Other recent studies have highlighted different sources of fibrosis-associated myofibroblasts. A perivascular mesenchymal stem cell-like population expressing the hedgehog transcriptional activator Gli1 is a myofibroblast source of solid organ and bone marrow fibrosis $(14,15)$. Inhibition of Gli1 directly or indirectly has therapeutic potential in targeting a particular pro-fibrotic myofibroblast population in organ-based fibrosis (16).

\section{Inflammation and the myofibroblast}

Inflammation can trigger a range of signalling pathways which ultimately result in tissue fibrosis. Both chronic inflammatory responses and the impairment of resolution of inflammatory cues can result in pro-fibrotic signalling.

Interleukin-6 (IL-6) is critical in the activation of fibroblasts in scleroderma (17). Taking advantage of the 'faSScinate' study in which scleroderma patients were treated with tocilizumab (an IL-6 receptor blocking antibody), Denton et al investigated the effects of IL-6 on fibroblast biology in vivo. They used explant dermal fibroblast cultures to demonstrate a profound effect of IL-6 on myofibroblast activation, highlighted the interactions between the TGF $\beta$ and IL- 6 pathways, proposed IL- 6 blockade 
as a way to regulate TGF $\beta$-induced fibrotic pathways in addition to inflammatory pathways and raised the interest in IL-6 antagonism as a possible treatment for scleroderma (17).

Increased Toll-like receptor $(T R)$ activation has been demonstrated during injury and in patients with persistent tissue fibrosis. Subsequently, a range of studies have identified ways to therapeutically target $T R R$ signalling. A novel function of the ubiquitin-editing cytosolic enzyme and major TLR negative regulator, A20, has been shown to attenuate TGF $\beta$ and lipopolysacharideinduced fibrotic responses. Thus, adiponectin, which upregulates A20 expression and reduces collagen expression, $\alpha \mathrm{SMA}$ and other pro-fibrotic gene expression by antagonising intracellular TGF $\beta /$ Smad signalling, has been proposed as a potential anti-fibrotic treatment (18). TLR4 activation is also affected by the EDA but not EDB splice variant of fibronectin , and a continuous exposure of resident fibroblasts to the EDA variant is thought to cause the persistent cutaneous fibrotic phenotype through a TLR4/TGF $\beta$ signalling cascade (19). Similarly to fibronectin, only one of the two splice variants of the glycoprotein tenascin-C has been positively correlated with SSc skin biopsies and TR R4 activation after RNA-Seq (19). Targeting specific splice variants of fibronectin and tenascinC leads to a reduction in fibrotic responses as T5342126, a compound which blocks association of TRR4 with its co-receptor MD2, was shown to prevent myofibroblast differentiation, collagen 1 stimulation and to reverse organ fibrosis in mice, highlighting the role of TRR4 in myofibroblast and fibrosis (20).

NLRP3 inflammasome-driven collagen production is another process that characterises persistent skin fibrosis. Interleukin 1 (IL-1)-induced miRNA-155 has been shown to modulate fibrosis by upregulating NLRP3 inflammasome-driven collagen production. Potential inhibitors of IL-1 signalling (i.e. Rilonacept), have thus been proposed as potential scleroderma therapies (21).

\section{Epigenetic effects on fibroblast activation}


Epigenetic regulation of several key wound healing processes and ECM components ensures tissue homeostasis (22). However, epigenetic alterations in patients with organ fibrosis, thought to be triggered by persistent inflammation and pro-fibrotic cytokine signalling, can promote myofibroblast phenotype and aid the initiation and establishment of fibrosis $(23,24)$.

Various epigenetic modifications, such as DNA methylation, histone modifications and microRNAs cause or enhance the fibrotic phenotype. Friend-leukemia Virus Intergation 1 (FL1) and Krüppel-like factor 5 (KLF5), are both epigenetically silenced due to their hypermethylation in scleroderma, while

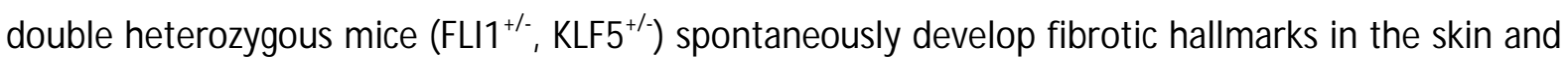
oesophagus, such as impaired collagen fibril structure, and characteristic cytokine and chemokine expression profiles (25). Fப1 downregulation has also been found to contribute to autoimmune response activation by activating autoimmune regulator (AIRE) expression in the thymus, connecting epigenetic modulation and the control of inflammatory processes during the fibrotic reaction (25). Methyl-CpG-binding protein 2 (MeCP2) has been identified as an anti-fibrotic epigenetic regulator, as it can inhibit fibroblast trans-differentiation, migration and proliferation as well as modulate profibrotic genes such as COL1A1 and $\alpha$-SMA. Mediators like NID2, PLAU and ADA can be regulated to overexpress MeCP2 in scleroderma fibroblasts and thereby attenuate pro-fibrotic responses (26). Pharmacological or genetic inactivation of Jumonji domain-containing protein 3 (JMJD3) - a histone demethylase with an increased TGF $\beta$-dependent expression in scleroderma - can also lead to antifibrotic effects on fibroblasts, mediated by the downregulation of the transcription factor FRA2 (27). Poly(ADP-ribose) polymerase-1 (PARP-1) negatively regulates the canonical TGF $\beta$ signalling cascade in skin fibroblasts by inhibiting TGF $\beta /$ Smad3 signalling. In SSc, TGF $\beta$ signalling silences PARP-1 by hypermethylating its promoter region, leading to enhanced skin fibroblast activation and collagen production. The use of small molecule inhibitors for DNA methyltrasnferases has already been approved for clinical use (i.e. 5-azacytidine) and could also be applied to de-methylate PARP-1 and facilitateblocking of TGF $\beta / S m a d$ signalling (28). 
Histone deacetylase (HDAC) inhibitors have also emerged as potential anti-fibrotic therapies. Scriptaid, a recently identified HDAC inhibitor, has shown anti-fibrotic properties through blocking TGF $\beta$-induced fibrotic responses in fibroblasts, reducing ECM deposition, contractility and stiffness (29). Furthermore, Akamata et al (27) have identified the mitochondrial deacetylase sirtuin3 (SIRT3) as a target for fibroblast activation in scleroderma and other fibrotic diseases (30). These authors demonstrated that SIRT3 has anti-fibrotic functions in skin and lung fibroblasts and these propertiesare impaired in scleroderma and using a novel SIRT3 agonist (hexafluoro) inhibits TGF $\beta$ induced responses in vitro and in vivo.

\section{Cell signalling and myofibroblast differentiation}

TGF $\beta$ signalling has long been established as an important driver of both myofibroblast differentiation in fibrosis and in scleroderma. Apart from the canonical TGF $\beta$-induced activation through SMADs and non-canonical activation involving MAPK, Rho, JNK, PI3KIAKT and Focal Adhesion Kinase (FAK) pathways, TGF $\beta$ orchestrates effects of other critical pathways such as the WNT/ $\beta$-catenin and Hippo pathways $(7,31)$ which have important roles in cell growth and survival metabolism, phenotypic modulation, epithelial-mesenchymal transition (EMT) and Endomesenchymal transition (EndoMT).

Recently, Wei et al investigated the role of the non-neuronal cyclin-dependent kinase (CDK5) and its activator p35 (CDK5R1) in persistent myofibroblast production and fibrosis in scleroderma (32). They demonstrated that CDK5 is up-regulated by TGF $\beta$ and expression is elevated in scleroderma dermal fibroblasts resulting in increased profibrotic gene expression. The specific CDK5 inhibitor Roscovitine, attenuated the myofibroblast phenotype and reduced collagen levels in scleroderma fibroblasts (32).

A new pathway involves the action of the protein tyrosine phosphatases PTP4Al, found to be overexpressed in scleroderma dermal fibroblasts, where PTP4A1 promotes TGF $\beta$ signalling by 
enhancing extracellular regulated kinase (ERK) activity and stimulating SMAD3. PTP4A1, which also interacts with and inhibits SRC kinase basal levels and activity, may be a selective therapeutic target for TGF $\beta$-induced fibrosis (33). Bosutinib, a third generation Src kinase inhibitor has also been tested in vivo, validating its anti-fibrotic effects as a result of Src/ c-Abl kinase suppression and their inability to control TGF $\beta$ signalling, resulting in a dose-dependent decrease in skin fibrosis, collagen deposition, hydroxyproline levels, connective tissue growth factor (CTGF) , ERK1 \& 2 as well as other pro-fibrotic genes (34).

Another novel signalling pathway was reported by Tomcik at al who demonstrated enhanced expression of the tribbles homologue 3 (TRB3) pseudokinase through TGF $\beta$ signalling to bind and activate Smad3 transcription factors in order to increase collagen secretion and induce a positive TGF $\beta /$ Smad3 signalling feedback loop to regulate fibroblast activation (35).

Aberrant metabolic processes that lead to the Warburg effect, mitochondrial dysfunction and oxidative stress, have been described in both fibrosis and scleroderma (36). In a recent study, Goodwin et al demonstrated how hypoxia induced glycolytic reprogramming promotes lung myofibroblast differentiation (37) and suggested that targeting glycolysis may be a therapeutic alternative for patients with pulmonary fibrosis.

\section{Responses to the EOM}

Extensive ECM deposition is another hallmark of fibrosis which has long been thought of as just the consequence of a fibrotic state. However, one fundamental aspect of pro-fibrotic environment is the regulation of fibroblast mechanoactivation towards the myofibroblastic phenotype, a process that is controlled largely by matrix stiffness (38). Fibroblasts can probe, interrogate and 'sense' the ECM by interactions with their integrin-bound focal adhesion complex. Fibroblast can then integrate these mechanical cues into specific intracellular signalling pathways depending on ECM stiffness. In a stiff fibrotic environment, myofibroblasts persist, producing and crosslinking ECM components 
excessively, through multiple feed-forward loops and mechanotransduction pathways which promote the establishment of fibrosis (39).

Many studies aim to dissect the mechanotransduction pathways in organ fibrosis and how the ECM micro-archecture is involved, in order to identify targets to develop novel therapeutic strategies.

The HIPPO pathway appears the only identified mechanosensing pathway that negatively regulates fibrosis by blocking Yes Associated Protein (YAP) nudear localisation via its phosphorylation and degradation. Liang et al have recently shown that myofibroblast trans-differentiation occurs as a result of YAP activation in response to altered ECM stiffness, forming a feed-forward loop resulting in kidney fibrosis through the Hippo mechanosensing pathway (40). The use of verteporfin in mouse unilateral ureteral obstruction (UUO) models was also found to prevent UUO-induced matrix deposition and fibrosis through blocking YAP/TEAD association and consequently nuclear localisation (40). Other studies have found that, bleomycin-induced skin fibrosis in mice was prevented with the use of dimethyl fumarate, where YAP/TAZ nuclear localisation is blocked through the inhibitory phosphorylation of phosphatidylinositol 3 kinase/protein kinase $B$ on the Akt/GSK3ß/YAP/TAZ mechanotransduction pathway (41). Downregulation of the Akt mechanosensing pathway has also been achieved through the use of selexipag and ACT-333679, to reduce expression and production of the EOM proteins collagen 1 and fibronectin, potentially interfering with the pro-fibrotic myofibroblast activity in scleroderma (42).

Collagen bundle alignment is another important characteristic of the fibrotic matrix and has recently been correlated to the directed cell migration of fibroblasts towards the ECM and inhibited cell division via the upregulation of Arhgdib (Rho GDP-dissociation inhibitor 2) (43).

Notably, the use of KW6002 a selective antagonist of the Adenosine $A_{2 a}$ Receptor which upon stimulation promotes the production of collagens 1 and 3, has been studied in the murine model of 
bleomycin-induced dermal fibrosis. KW6002 reduced skin thickness, myofibroblast accumulation and collagen bundle alignment, through crosstalk with the WNT/ $\beta$-catenin signalling pathway (44).

Finally, stiff matrix cell stimulation has been shown to block myofibroblast apoptosis and enhance the fibrotic phenotype through the FAK/YAP/TAZ/BCXL mechanotransduction pathway that affects the expression of the BC-XL (B-cell lymphoma-extra large) gene (45). As a result, it was shown that apoptosis pathways can be re-activated through interrupting mechanotransduction signals using navitodax (ABT-263), a drug which blocks $B C-X \mathbb{L}$ action leading to a reduction of fibrosis in bleomycin mice (45).

\section{Condusion}

Myofibroblasts are prevalent in most organs. They are metabolically active, contractile, regulate connective tissue homeostasis and are pivotal coordinators of tissue repair. The identification of mesenchymal progenitor populations and fibroblast heterogeneity suggests myofibroblasts may arise from diverse developmental origins. In fibrosis, critical changes in myofibroblast phenotype and function, such as matrix production, growth factor secretion and increased survival and persistence are regulated by aspects of inflammation, epigenetic modifiers, cell signalling and ECM composition and architecture alterations. Recent studies delineating the key regulatory pathways and networks involved have revealed potential new and innovative targets for improved and effective treatment of fibrosis.

\section{Key Points}

- Recent studies strongly suggest that the origins of myofibroblast may reside in diverse cell populations and progenitors cell types.

- Resident dermal fibroblast are highly heterogeneous cell populations with distinct development origins and exhibiting different intrinsic transcriptional programmes and functional properties in tissue maintenance, repair and fibrosis 
- Exciting data highlight the role of several epigenetic regulatory pathways in governing the extent, tempo and severity of tissue fibrosis

- ECM stiffness and biomechanical cues are critical drivers and regulators of fibrosis and there are specific cell signalling pathways that are involved in sensing the extracellular microenvironment

- New studies focus on distinct, selective and innovative treatment modalities for fibrosis centred around evidence-based pathobiology targeting aspects of inflammation, cell signalling pathways, epigenetics regulators/modulators and critical interactions with the stromal environment.

\section{Adknowledgements}

None

\section{Finandial Support and sponsorship}

This work was supported by the Centre of Rheumatology and Connected Tissue Diseases, University College London, London, UK. We could acknowledge Arthritis Research UK, Scleroderma Research UK, the Rosetrees Trust and the Royal Free Charity.

\section{Conflicts of interest}

None

\section{Figurelegands}

\section{Fig1 Myofibroblast activation}

Key factors affecting the establishment of myofibroblasts include the origin of subpopulations within tissues, epigenetic regulation of critical cellular processes involved in tissue remodelling such as inflammatory responses and cell interactions with ECM and its biomechanical properties. 
Myofibroblasts modify the ECM and can themselves induce alterations in these processes which result in persistent and sustained activation (red arrows). 


\section{References and recommended reading}

1. Hinz B. The role of myofibroblasts in wound healing. Curr Res Transl Med [Internet]. 2016 Oct [cited 2018 Jun 6];64(4):171-7. Available from: http://www.ndbi.nlm.nih.gov/pubmed/27939455

2. Pakshir P, Hinz B. The big five in fibrosis: Macrophages, myofibroblasts, matrix, mechanics, and miscommunication. Matrix Biol [Internet]. 2018Jan 31 [cited 2018 Jun 6]; Available from: http://www.ndbi.nlm.nih.gov/pubmed/29408013

** A comprehensive review of myofibroblast behaviour and function. This review highlights the role of the inflammatory macophage, biochemical signals and cell-to-cell / cell-to-matrix communication important in myofibroblast behaviour in tissue repair and fibrosis.

3. Denton CP, Khanna D. Systemic sclerosis. Lancet [Internet]. 2017 Oct 7 [cited 2018 Jun 6];390(10103):1685-99. Available

from:

https://www.sciencedirect.com/science/article/pii/S0140673617309339

*A comprehensive review of the scleroderma disease entity, covering advances in understanding autoimmunity, vasculopathy and fibrosis, and treatments for specific disease complications and future developments.

4. Lafyatis R. Transforming growth factor $\beta$-at the centre of systemic sclerosis. Nat Rev Rheumatol [Internet]. 2014 Dec 19 [cited 2018 Jun 6];10(12):706-19. Available from: http://www.ncbi.nlm.nih.gov/pubmed/25136781

5. Bayreuther K, Rodemann HP, Hommel R, Dittmann K, Albiez M, Francz PI. Human skin fibroblasts in vitro differentiate along a terminal cell lineage. Proc Natl Acad Sci U S A [Internet]. 1988 Jul [cited 2018 Jun 12];85(14):5112-6. Available from: http://www.ncbi.nlm.nih.gov/pubmed/3393534

6. Garret SM, Frost DB, Feghali-Bostwick C. The mighty fibroblast and its utility in scleroderma research. J scleroderma Relat Disord [Internet]. 2017 [cited 2018 Jun 6];2(2):69-134. Available from: http://www.ncbi.nlm.nih.gov/pubmed/29270465

7. Pardali E, Sanchez-Duffhues G, Gomez-Puerto MC, Ten Dijke P. TGF- $\beta$-Induced EndothelialMesenchymal Transition in Fibrotic Diseases. Int J Mol Sci [Internet]. 2017 Oct 17 [cited 2018 Jun 8];18(10). Available from: http:// www.ncbi.nlm.nih.gov/pubmed/29039786

8. Marangoni RG, Korman BD, Wei J, Wood TA, Graham LV, Whitfield ML, et al. Myofibroblasts in murine cutaneous fibrosis originate from adiponectin-positive intradermal progenitors. Arthritis Rheumatol (Hoboken, NJ) [Internet]. 2015 Apr [cited 2018 Jun 8];67(4):1062-73. Available from: http://www.ncbi.nlm.nih.gov/pubmed/25504959

9. Driskell RR, Lichtenberger BM, Hoste E, Kretzschmar K, Simons BD, Charalambous M, et al. Distinct fibroblast lineages determine dermal architecture in skin development and repair. Nature [Internet]. 2013 Dec 12 [cited 2018 Jun 6];504(7479):277-81. Available from: http://www.ncbi.nlm.nih.gov/ pubmed/24336287

** A seminal paper using lineage tracking and phenotypic markers to identify and define fibroblast heterogeneity in the skin and the subpopulations involved in repair.

10. Rinkevich Y, Walmsley GG, Hu MS, Maan $\mathbb{Z N}$, Newman AM, Drukker M, et al. Identification 
and isolation of a dermal lineage with intrinsic fibrogenic potential. Science (80- ) [Internet]. 2015 Apr 17 [cited 2018 Jun 14];348(6232):aaa2151-aaa2151. Available from: http://www.ncbi.nlm.nih.gov/pubmed/25883361

** Important and elegant in vivo study using developmental genetic markers to identify an intrinsic population of C26 (DPP4; dipeptidyl peptidase-4) positive skin fibroblasts with pro-fibrotic properties important for wound healing and fibrosis.

11. Hu MS, Longaker MT. Dipeptidyl Peptidase-4, Wound Healing, Scarring, and Fibrosis. Plast Reconstr Surg [Internet]. 2016 Nov [cited 2018 Jun 6];138(5):1026-31. Available from: http://www.ncbi.nlm.nih.gov/ pubmed/27782998

12. Tabib T, Morse C, Wang T, Chen W, Lafyatis R. SFRP2/DPP4 and FMO1/LSP1 Define Major Fibroblast Populations in Human Skin. J Invest Dermatol [Internet]. 2018 Apr [cited 2018 Jun 6];138(4):802-10. Available from: http://www.ncbi.nlm.nih.gov/pubmed/29080679

* An in depth study using single-cell RNA Seq to delineate and study dermal fibroblast populations and provides evidence to suggest there are distinct roles in ECM production, matrix deposition, inflammation, inflammation and differentiation.

13. Philippeos C, Telerman SB, Oulès B, Pisco AO, Shaw T], Elgueta R, et al. Spatial and Single-Cell Transcriptional Profiling Identifies Functionally Distinct Human Dermal Fibroblast Subpopulations. J Invest Dermatol [Internet]. 2018 Apr [cited 2018 Jun 6];138(4):811-25. Available from: http://www.ncbi.nlm.nih.gov/pubmed/29391249

14. Kramann R, Schneider RK, DiRocco DP, Machado F, Fleig S, Bondzie PA, et al. Perivascular Gli1+ Progenitors Are Key Contributors to Injury-Induced Organ Fibrosis. Cell Stem Cell [Internet]. 2015 Jan 8 [cited 2018 Jun 6];16(1):51-66. Available from: http://www.ncbi.nlm.nih.gov/pubmed/25465115

15. Schneider RK, Mullally A, Dugourd A, Peisker F, Hoogenboezem R, Van Strien PMH, et al. Gli1 + Mesenchymal Stromal Cells Are a Key Driver of Bone Marrow Fibrosis and an Important Cellular Therapeutic Target. Cell Stem Cell [Internet]. 2017 Jun 1 [cited 2018 Jun 6];20(6):785-800.e8. Available from: http://www.ncbi.nlm.nih.gov/pubmed/28457748

16. Kramann R, Schneider RK The identification of fibrosis-driving myofibroblast precursors reveals new therapeutic avenues in myelofibrosis. Blood [Internet]. 2018 May 10 [cited 2018 Jun 6];131(19):2111-9. Available from: http://www.ncbi.nlm.nih.gov/pubmed/29572380

17. Denton $\mathrm{CP}$, Ong VH, Xu S, Chen-Harris H, Modrusan Z, Lafyatis R, et al. Therapeutic interleukin-6 blockade reverses transforming growth factor-beta pathway activation in dermal fibroblasts: insights from the faSscinate clinical trial in systemic sclerosis. Ann Rheum Dis [Internet]. 2018 May 31 [cited 2018 Jun 6];annrheumdis-2018-213031. Available from: http://www.ncbi.nlm.nih.gov/pubmed/29853453

*Investigation of the effects of IL-6 and IL-6 antagonism (IL-6 receptor antibody; tocilizumab) on fibroblast biology in vivo as part of the 'faSscinate' study in which scleroderma patients were treated with tocilizumab. Based on these data, a phase 3 trial of tocilizumab is under way.

18. Bhattacharyya S, Wang W, Graham LVD, Varga J. A20 suppresses canonical Smad-dependent fibroblast activation: novel function for an endogenous inflammatory modulator. Arthritis Res Ther [Internet]. 2016 [cited 2018 Jun 6];18(1):216. Available from: http://www.ncbi.nlm.nih.gov/pubmed/27716397

19. Bhattacharyya S, Midwood KS, Yin H, Varga J. Toll-Like Receptor-4 Signaling Drives Persistent 
Fibroblast Activation and Prevents Fibrosis Resolution in Scleroderma. Adv Wound Care [Internet]. 2017 Oct 1 [cited 2018 Jun 6];6(10):356-69. Available from: http://www.ncbi.nlm.nih.gov/pubmed/29062592

** An excelent article following on from previous pioneering studies (in STM/Nat Comms) exploring the role of the TRRs and endogenous matrix ligands in scleroderma and defining Toll-Like Receptor-4 signalling as a key driver of persistent fibroblast activation (myofibroblasts) and a failure to resolve chronic fibrosis in scleroderma.

20. Bhattacharya S, Tamaki Z, Wang W, Hinchdiff M, Hoover P, Getsios S, et al. FibronectinEDA Promotes Chronic Cutaneous Fibrosis Through Toll-Like Receptor Signaling. Sci Transl Med [Internet]. 2014 Apr 16 [cited 2018 Jul 9];6(232):232ra50-232ra50. Available from: http://www.ndbi.nlm.nih.gov/pubmed/24739758

21. Artlett CM, Sassi-Gaha S, Hope JL, Feghali-Bostwick CA, Katsikis PD. Mir-155 is overexpressed in systemic sclerosis fibroblasts and is required for NLRP3 inflammasome-mediated collagen synthesis during fibrosis. Arthritis Res Ther [Internet]. 2017 Dec 17 [cited 2018 Jun 6];19(1):144. Available from: http://www.ncbi.nlm.nih.gov/pubmed/28623945

22. Piperigkou Z, Götte $M$, Theocharis $A D$, Karamanos NK. Insights into the key roles of epigenetics in matrix macromolecules-associated wound healing. Adv Drug Deliv Rev [Internet]. 2017 Oct 24 [cited 2018 Jun 6]; Available from: http://www.ndbi.nlm.nih.gov/pubmed/29079535

23. Bergmann C, Distler JH. Epigenetic factors as drivers of fibrosis in systemic sclerosis. Epigenomics [Internet]. 2017 Apr [cited 2018 Jun 6];9(4):463-77. Available from: http://www.ncbi.nlm.nih.gov/ pubmed/28343418

24. Yang J, Tian B, Brasier AR. Targeting Chromatin Remodeling in Inflammation and Fibrosis. In: Advances in protein chemistry and structural biology [Internet]. 2017 [cited 2018 Jun 6]. p. 136. Available from: http://www.ncbi.nlm.nih.gov/pubmed/28215221

25. Takahashi T, Asano Y, Sugawara K, Yamashita T, Nakamura K, Saigusa R, et al. Epithelial Fli1 deficiency drives systemic autoimmunity and fibrosis: Possible roles in scleroderma. J Exp Med [Internet]. 2017 Apr 3 [cited 2018 Jun 6];214(4):1129. Available from: http://www.ncbi.nlm.nih.gov/pubmed/28232470

26. He Y, Tsou P-S, Khanna D, Sawalha AH. Methyl-CpG-binding protein 2 mediates antifibrotic effects in scleroderma fibroblasts. Ann Rheum Dis [Internet]. 2018 May 14 [cited 2018 Jun 6];annrheumdis-2018-213022. Available from: http://www.ndbi.nlm.nih.gov/pubmed/29760157

* Informative article reporting evidence of epigenetic alteration in scleroderma and the dysregulation of histone demethylase modulating fibroblast activation by regulating the levels of histone methylation on transcription factor promoters. This study highlights modulation of histone demethylase as a potentially antifibrotic approach in scleroderma.

27. Bergmann C, Brandt A, Merlevede B, Hallenberger L, Dees C, Wohlfahrt T, et al. The histone demethylase Jumonji domain-containing protein 3 (JMJD3) regulates fibroblast activation in systemic sclerosis. Ann Rheum Dis [Internet]. 2018 Jan [cited 2018 Jun 6];77(1):150-8. Available from: http://www.ncbi.nlm.nih.gov/pubmed/29070530

28. Zhang Y, Pötter S, Chen C-W, Liang R, Gelse K, Ludolph I, et al. Poly(ADP-ribose) polymerase-1 regulates fibroblast activation in systemic sclerosis. Ann Rheum Dis [Internet]. 2018 May 1 [cited 2018 Jun 7];77(5):744-51. Available from: 
http://www.ndbi.nlm.nih.gov/pubmed/29431122

29. Kim DJ, Dunleavey JM, Xiao L, Ollila DW, Troester MA, Otey CA, et al. Suppression of TGFßmediated conversion of endothelial cells and fibroblasts into cancer associated (myo)fibroblasts via HDAC inhibition. Br J Cancer [Internet]. 2018 May 26 [cited 2018 Jun 6];118(10):1359-68. Available from: http://www.ncbi.nlmnih.gov/pubmed/29695769

30. Akamata K, Wei J, Bhattacharyya M, Cheresh P, Bonner MY, Arbiser JL et al. SIRT3 is attenuated in systemic sclerosis skin and lungs, and its pharmacologic activation mitigates organ fibrosis. Oncotarget [Internet]. 2016 Oct 25 [cited 2018 Jun 8];7(43):69321-36. Available from: http://www.ncbi.nlm.nih.gov/pubmed/27732568

31. Lecarpentier Y, Schussler O, Claes V, Vallée A. The Myofibroblast: TGF $\beta-1$, A Conductor which Plays a Key Role in Fibrosis by Regulating the Balance between PPARY and the Canonical WNT Pathway. Nucl Recept Res [Internet]. 2017 [cited 2018 Jun 8];4. Available from: http://www.agialpress.com/journals/nurr/2017/101299/

32. Wei J, Marangoni RG, Fang F, Wang W, Huang J, Distler JHW, et al. The non-neuronal cyclindependent kinase 5 is a fibrotic mediator potentially implicated in systemic sclerosis and a novel therapeutic target. Oncotarget [Internet]. 2018 Feb 13 [cited 2018 Jun 6];9(12):10294306. Available from: http://www.ncbi.nlmnih.gov/pubmed/29535807

33. Sacchetti C, Bai Y, Stanford SM, Di Benedetto P, Gpriani P, Santelli E, et al. PTP4A1 promotes TGF $\beta$ signaling and fibrosis in systemic sclerosis. Nat Commun [Internet]. 2017 Dec 20 [cited 2018Jun 8];8(1):1060. Available from: http://www.nature.com/artides/s41467-017-01168-1

34. Wermuth PJ, Jimenez SA. Abrogation of transforming growth factor- $\beta$-induced tissue fibrosis in TBRIcaColla2Cre transgenic mice by the second generation tyrosine kinase inhibitor SKI606 (Bosutinib). Gullberg D, editor. PLoS One [Internet]. 2018 May 2 [cited 2018 Jun 8];13(5):e0196559. Available from: http://dx.plos.org/10.1371/journal.pone.0196559

35. Tomcik M, Palumbo-Zerr K, Zerr P, Sumova B, Avouac J, Dees C, et al. Tribbles homologue 3 stimulates canonical TGF- $\beta$ signalling to regulate fibroblast activation and tissue fibrosis. Ann Rheum Dis [Internet]. 2016 Mar [cited 2018 Jun 7];75(3):609-16. Available from: http://www.ncbi.nlm.nih.gov/pubmed/25603829

36. Murgia F, Svegliati S, Poddighe S, Lussu M, Manzin A, Spadoni T, et al. Metabolomic profile of systemic sclerosis patients. Sci Rep [Internet]. 2018 Dec 16 [cited 2018 Jun 6];8(1):7626. Available from: http://www.ncbi.nlm.nih.gov/pubmed/29769578

37. Goodwin J, Choi H, Hsieh M, Neugent ML, Ahn J-M, Hayenga HN, et al. Targeting HypoxiaInducible Factor-1 $\alpha /$ Pyruvate Dehydrogenase Kinase 1 Axis by Dichloroacetate Suppresses Bleomycin-induced Pulmonary Fibrosis. Am J Respir Cell Mol Biol [Internet]. 2018 Feb [cited 2018 Jun 6];58(2):216-31. Available from: http://www.atsjournals.org/ doi/10.1165/rcmb.2016-01860C

38. Santos A, Lagares D. Matrix Stiffness: the Conductor of Organ Fibrosis. Curr Rheumatol Rep [Internet]. 2018 Jan 19 [cited 2018 Jun 6];20(1):2. Available from: http://www.ncbi.nlm.nih.gov/pubmed/29349703

39. Schulz J-N, Plomann M, Sengle G, Gullberg D, Krieg T, Eckes B. New developments on skin fibrosis - Essential signals emanating from the extracellular matrix for the control of myofibroblasts. Matrix Biol [Internet]. 2018 Feb 12 [cited 2018 Jun 6]; Available from: http://www.ncbi.nlm.nih.gov/pubmed/29408278 
40. Liang M, Yu M, Xia R, Song K, Wang J, Luo J, et al. Yap/Taz Deletion in Gli ${ }^{+}$Cell-Derived Myofibroblasts Attenuates Fibrosis. J Am Soc Nephrol [Internet]. 2017 Nov [cited 2018 Jun 6];28(11):3278-90. Available from: http://www.ndbi.nlmnih.gov/pubmed/28768710

* This article repors studies targetng components of the 'Hippo pathway' in scleroderma using a small molecule metabolite dimethyl fumarate (DMF). Investigations link the influence of DMF to blockade of TGF $\beta$ signalling and translocation of YAP/TAZ, and suggest a role for DMF in rtreating fibrosis is scleroderma. Interestingly, DMF also modulated proteasomal degradation and enhanced the antioxidant/anti-inflammatory response via activation of Nrf2.

41. Toyama T, Looney AP, Baker BM, Stawski L, Haines P, Simms R, et al. Therapeutic Targeting of TAZ and YAP by Dimethyl Fumarate in Systemic Sclerosis Fibrosis. J Invest Dermatol [Internet]. 2018 Jan [cited 2018 Jun 6];138(1):78-88. Available from: http://www.ndbi.nlm.nih.gov/pubmed/28870693

42. Cutolo M, Ruaro B, Montagna P, Brizzolara R, Stratta E, Trombetta AC, et al. Effects of selexipag and its active metabolite in contrasting the profibrotic myofibroblast activity in cultured scleroderma skin fibroblasts. Arthritis Res Ther [Internet]. 2018 May 2 [cited 2018 Jun 6];20(1):77. Available from: http://www.ncbi.nlm.nih.gov/pubmed/29720235

43. Cao L, Lafyatis R, Burkly LC. Increased dermal collagen bundle alignment in systemic sclerosis is associated with a cell migration signature and role of Arhgdib in directed fibroblast migration on aligned ECMs. Gullberg D, editor. PLoS One [Internet]. 2017 Jun 29 [cited 2018 Jun 6];12(6):e0180751. Available from: http://www.ncbi.nlm.nih.gov/pubmed/28662216

44. Zhang J, Corciulo C, Liu H, Wilder T, Ito M, Cronstein B. Adenosine A 2a Receptor Blockade Diminishes Wnt/ $\beta$-Catenin Signaling in a Murine Model of Bleomycin-Induced Dermal Fibrosis. Am J Pathol [Internet]. 2017 Sep [cited 2018 Jun 6];187(9):1935-44. Available from: http://www.ncbi.nlm.nih.gov/ pubmed/28667836

45. Lagares D, Santos A, Grasberger PE, Liu F, Probst CK, Rahimi RA, et al. Targeted apoptosis of myofibroblasts with the BH3 mimetic ABT-263 reverses established fibrosis. Sci Transl Med [Internet]. 2017 Dec 13 [cited 2018 Jun 6];9(420):eaal3765. Available from: http://www.ncbi.nlm.nih.gov/pubmed/29237758

**Important study focusing on myofibroblast programmed cell death, and demonstrating that inhibition of apoptosis enhances the responses of myofibroblast to EOM and highlights the role of mechanosensing in persistent myofibroblast activation. 


\begin{tabular}{|c|c|c|c|c|c|}
\hline Adipocytes & $\begin{array}{l}\text { Bone Marrow } \\
\text { Derived progenitor } \\
\& \text { stem cells }\end{array}$ & $\left\{\begin{array}{l}\text { Endothelial \& } \\
\text { Epithelial cells }\end{array}\right.$ & $\begin{array}{l}\text { Tissue Resident } \\
\text { Fibroblast populations }\end{array}$ & $\begin{array}{l}\text { Pericytes \& } \\
\text { Vascular Smooth } \\
\text { Muscle Cells }\end{array}$ & $\begin{array}{l}\text { Resident-mesenchymal } \\
\text { progenitor cells }\end{array}$ \\
\hline
\end{tabular}

Origin \& Sources

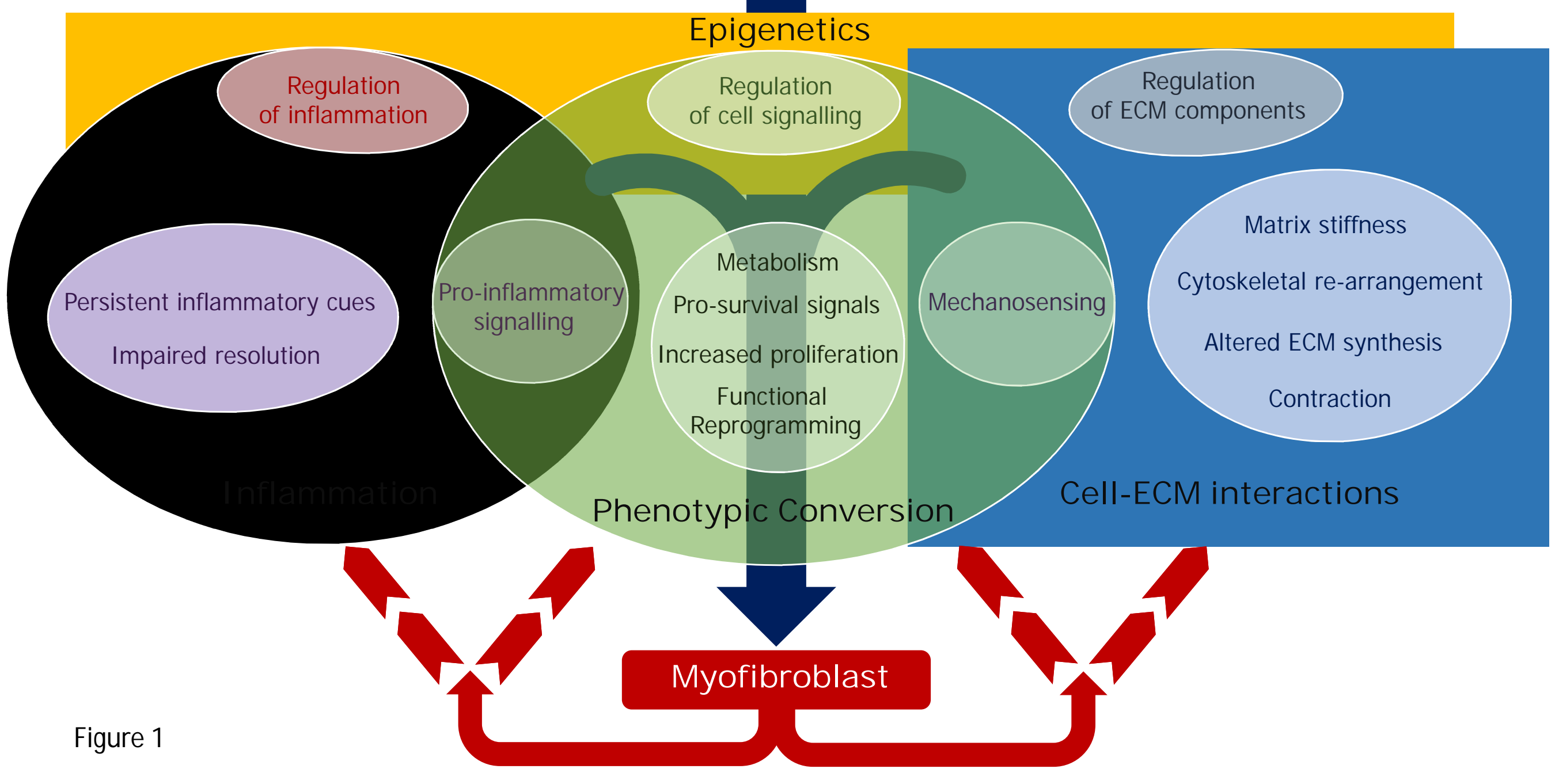


